\title{
Carotid intima-media thickness in the Brazilian Longitudinal Study of Adult Health (ELSA-Brasil): a narrative review
}

\author{
Eduardo Henrique Sena Santos', Pedro José dos Santos Neto', Itamar de Souza Santos"
}

Hospital Universitário (HU), Universidade de São Paulo (USP), São Paulo (SP), Brazil

\begin{abstract}
'MD. Physician, Imaging Service, and Researcher, Center for Clinical and Epidemiological Research, Hospital Universitário (HU), Universidade de São Paulo (USP), São Paulo (SP), Brazil.

"MD, PhD. Researcher, Center for Clinical and Epidemiological Research, Hospital Universitário (HU), Universidade de São Paulo (USP), and Associate Professor, Department of Internal Medicine, Faculdade de Medicina da Universidade de São Paulo (FMUSP), São Paulo (SP), Brazil.

(Dorcid.org/0000-0003-3212-8466
\end{abstract}

\section{KEY WORDS:}

Carotid intima-media thickness.

Atherosclerosis.

Cardiovascular system.

Risk factors.

Cross-sectional studies.

\begin{abstract}
BACKGROUND: Carotid intima-media thickness (CIMT), as measured by ultrasound, has been used in large studies as a non-invasive marker for subclinical atherosclerosis. The Brazilian Longitudinal Study of Adult Health (ELSA-Brasil) is a cohort of 15,105 civil servants in six Brazilian cities that included CIMT evaluation in its baseline assessment. The aim of the present narrative review was to provide an overview of ELSA-Brasil CIMT articles published up to July 31, 2017.

DESIGN AND SETTING: Narrative review of ELSA-Brasil CIMT studies using baseline assessment data. METHODS: We searched PubMed for the terms "ELSA-Brasil" and "intima-media". This search yielded 21 published articles using CIMT data from the ELSA-Brasil baseline assessment, which were included in this review. We also present information about intima-media thickness assessment from ongoing onsite reevaluations of the study participants.

RESULTS: Most published studies focused on the association with traditional and novel cardiovascular risk factors. Studies also presented information about the ELSA-Brasil CIMT protocol at baseline and CIMT value distribution in this large sample.

CONCLUSIONS: Analyses on the ELSA-Brasil data led to important insights on CIMT interpretation and physiology. Besides the highlighted contributions which have already been made in this field, new data gathered during the ongoing third onsite assessment will enable investigation of substantially new research questions.
\end{abstract}

\section{INTRODUCTION}

Intima-media thickness (IMT), as measured by ultrasound, has been used in both observational and intervention studies as a non-invasive marker for subclinical atherosclerosis. ${ }^{1}$ It has been shown to be associated with cardiovascular risk factors, ${ }^{2}$ and to be a predictor of cardiovascular events. ${ }^{3}$ Carotid IMT (CIMT) progression over time is used as a surrogate outcome for atherosclerotic disease in clinical trials. ${ }^{4}$

Ultrasound examination allows adequate characterization of carotid walls, is easily executed, is less costly than other imaging techniques, and is widely available. CIMT can be measured by means of B-mode ultrasound, and is defined as the distance between two interfaces: (a) the vascular and intima lumina; and (b) the middle and adventitial layers. ${ }^{1}$ Despite many efforts that have been made towards standardizing CIMT definitions, there is no complete consensus in the literature about the best anatomical location, the number of measurements, or which summary of measurements (mean or maximum CIMT) should be used.

The Brazilian Longitudinal Study of Adult Health (ELSA-Brasil) ${ }^{5,6}$ is a cohort study on 15,105 civil servants aged 35 to 74 years, in six Brazilian cities (São Paulo, Belo Horizonte, Rio de Janeiro, Porto Alegre, Salvador and Vitória). CIMT data were collected at baseline between August 2008 and December 2010. ELSA-Brasil has one of the largest CIMT datasets among observational studies. As a reference, the ELSA-Brasil CIMT sample size is comparable to large, iconic cohorts that included CIMT evaluation, such as the Atherosclerotic Risk in Communities (ARIC) ${ }^{7}$ study and the Multi-Ethnic Study of Atherosclerosis (MESA), ${ }^{8}$ and is larger than other important CIMT samples such as the Northern Manhattan (NOMAS) ${ }^{9}$ and Rotterdam ${ }^{10}$ studies.

The aim of the present narrative review was to provide an overview of articles on CIMT in ELSA-Brasil cohort articles published prior to July 31, 2017, and to summarize the contributions that these studies have made to current knowledge. 


\section{METHODS}

We searched PubMed for the terms "ELSA-Brasil" and "intimamedia" to select the articles included in this review. ELSA-Brasil has a steering committee and a publications and presentations committee. ${ }^{11}$ Both are composed of researchers from each ELSABrasil investigation site and also keep track of these published papers, which were added to this review.

We classified these articles according to their main objectives, into three subsections: "CIMT protocol and value distributions at ELSA-Brasil baseline”, "CIMT and traditional cardiovascular risk factors" and "CIMT and novel cardiovascular risk factors or other conditions". We also present information about IMT assessment from ongoing onsite reevaluations of study participants (ELSABrasil Visit 3) and some of the study perspectives for the near future.

\section{RESULTS}

We found 21 published articles using CIMT data from the baseline assessment. Table $\mathbf{1}^{12-20}$ shows the main aims, findings and conclusions of articles focusing on the association with traditional risk factors and Table $2^{21-32}$ shows the main aims, findings and conclusions of articles focusing on the association with novel cardiovascular risk factors or other conditions.

\section{DISCUSSION}

\section{CIMT protocol and value distributions at ELSA-Brasil baseline}

Acquisition of CIMT images in the ELSA-Brasil study complied with the recommendations from the American and Brazilian Societies of Echocardiography. All patients were examined by technicians and/or physicians who had previously been trained and certified for this protocol. The images were obtained using Toshiba Aplio XG ultrasound devices with a linear 7.5 MHz transducer. All images obtained were sent to an examination reading center in São Paulo. To measure CIMT, each common carotid artery (CCA) was identified along its longitudinal axis, using standard brightness and contrast. IMT was calculated automatically using the Medical Imaging Applications software (MIA, Coralville, Iowa, USA), with analysis on three electrocardiographically gated cardiac cycles. Only the proximal far wall of the CCA $(1 \mathrm{~cm}$ proximally to the carotid bifurcation, with $1 \mathrm{~cm}$ length) was measured, and mean and maximum values for each CCA were obtained. ${ }^{33}$

The current Brazilian recommendations for ultrasound evaluation on carotid atherosclerotic disease use the ELSA-Brasil CIMT values as the standard for the Brazilian adult population. ${ }^{34}$ These standards are important, because a CIMT value $\geq$ the $75^{\text {th }}$ percentile for an individual's age, sex and race/ethnicity, and which has been determined from the distribution in his/her reference population, is usually considered indicative of higher subclinical atherosclerosis burden. ${ }^{35}$
Comparing IMT values across cohorts is difficult. Differences in study populations, sample selection, year of recruitment, CIMT protocols and statistical choices influence these comparisons. Considering the context of these limitations, Santos et al. ${ }^{12}$ showed that for the same sex, age and race, the CIMT values in ELSA-Brasil were on the whole slightly lower than those in the ARIC study, but were similar to the findings from the German Gutenberg Heart Study.

\section{CIMT and traditional cardiovascular risk factors}

The abovementioned article by Santos et al. ${ }^{12}$ also assessed some important associations for the Brazilian population. A considerable proportion (43\%) of Brazilians reported themselves as "mixed race" in the 2010 national census. This high rate of racial blending, which is also reflected in the ELSA-Brasil sample, cannot be found in other large CIMT samples. These authors concluded that black individuals had higher CIMT values than did white or mixed-race individuals. CIMT values for individuals who self-reported their race as mixed were closer to those found for whites than to those for blacks, after adjustment for age and sex. These findings may reflect constitutional aspects of this measurement, but may also be a marker for racial health inequalities.

New insights about the interpretation of CIMT values came from a 2015 ELSA-Brasil article by Santos et al. ${ }^{20}$ The authors found that traditional cardiovascular risk factors explained less than $40 \%$ of CIMT variance in the ELSA-Brasil sample, even after adopting analytical strategies to optimize this proportion. This finding is in line with results from other samples, and suggests that there is room to analyze novel risk factors for atherosclerosis in determining CIMT values. It is possible that other factors that are not directly linked to atherosclerosis progression may influence CIMT values. For example, some of these factors may be associated with medial hypertrophy, a condition that may increase CIMT values as well. Identification of these additional factors that are unrelated to atherosclerosis is important for understanding CIMT and its association with cardiovascular risk.

Additional analyses investigating the association between CIMT values and cardiovascular health $(\mathrm{CVH})$ scores at the ELSA-Brasil baseline have corroborated these interpretations. The $\mathrm{CVH}$ score was proposed by the American Heart Association in $2010^{36}$ as a tool for measuring $\mathrm{CVH}$ in populations. It has well-defined criteria for diet, physical activity, body mass index, smoking, blood pressure, fasting plasma glucose and total cholesterol score. Significant inverse associations were found between $\mathrm{CVH}$ scores and CIMT values at the ELSA-Brasil baseline, although some participants with optimum CVH scores had unexpectedly high CIMT values. ${ }^{13}$

Another finding from the ELSA-Brasil data was the close relationship between CIMT values and neck circumference (NC). ${ }^{17}$ $\mathrm{NC}$ was associated with CIMT values but not with coronary artery 
calcium, an alternative marker for subclinical atherosclerosis. Moreover, after adjustment for CCA vessel diameters, this association remained significant, thus suggesting that this does not merely result from larger CCA arteries in individuals with greater NC. One putative explanation for this finding is that there may be a paracrine effect from fatty tissue in the neck, which would influence carotid atherosclerosis but would not have an impact on arteries in other regions.
An association between CIMT values and blood pressure (BP) has been described in the ELSA-Brasil cohort ${ }^{20}$ and in other samples. ${ }^{37}$ Another two ELSA-Brasil articles have explored additional aspects of this association. Lotufo et al. ${ }^{18}$ analyzed data from ELSABrasil participants who were using antihypertensive medications. These authors found that those with resistant hypertension (defined as non-controlled BP despite using at least three different classes of antihypertensive medication or controlled BP through using at least

Table 1. ELSA-Brasil studies evaluating the association between carotid intima-media thickness and traditional cardiovascular risk factors

\begin{tabular}{|c|c|}
\hline Main aims and associations & Main findings \\
\hline Age, sex and race ${ }^{12}$ & 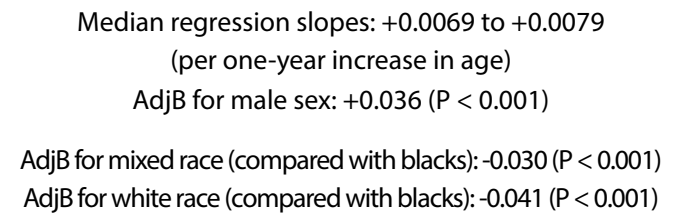 \\
\hline AHA CVH score ${ }^{13}$ & $\begin{array}{l}\text { AdjB for one-point increase in the CVH score: }-0.011 \\
\qquad(95 \% \mathrm{Cl}:-0.012 \text { to }-0.010)\end{array}$ \\
\hline Blood pressure variability ${ }^{14}$ & $\begin{array}{l}\text { Standardized path coefficient from BP variability } \\
\text { to residual CIMT (after regression to assess main } \\
\text { confounders): }+0.046(P<0.001)\end{array}$ \\
\hline $\begin{array}{l}\text { Glucose levels } 10 \text { to } 12 \text { years } \\
\text { before baseline }\end{array}$ & $\begin{array}{l}\text { AdjB for glucose levels } 110-125 \mathrm{mg} / \mathrm{dl}:+0.028 \\
\qquad(95 \% \mathrm{Cl}:+0.003 \text { to }+0.053) \\
\text { AdjB for incident diabetes: }+0.034 \\
(95 \% \mathrm{Cl}:+0.015 \text { to }+0.053)\end{array}$ \\
\hline HALP ${ }^{16}$ & AdjB for HALP: -0.020 (95\% Cl: -0.042 to +0.003$)$ \\
\hline Neck circumference $^{17}$ & $\begin{array}{c}\text { AdjOR for the association between one SD increase in NC } \\
\text { and CIMT } \geq 75^{\text {th }} \text { percentile } \\
\text { Men: } 1.66(95 \% \mathrm{Cl}: 1.28-2.14) \\
\text { Women: } 1.52 \text { (95\% Cl: } 1.16-1.99)\end{array}$ \\
\hline Resistant hypertension ${ }^{18}$ & $\begin{array}{l}\text { Prevalence ratio for resistant hypertension associated } \\
\text { with higher CIMT values: } 3.15 \text { (95\% Cl: } 2.09-4.74)\end{array}$ \\
\hline Smoking ${ }^{19}$ & $\begin{array}{l}\text { AdjB for current smokers: }+0.03(95 \% \mathrm{Cl}:+0.02 \text { to }+0.04) \\
\text { AdjB for former smokers: }+0.01(95 \% \mathrm{Cl}:+0.01 \text { to }+0.02) \\
\text { Interaction between age and current smoking: }+0.013(\mathrm{P}<0.001) \\
\text { Interaction between female sex and current smoking: } \\
\quad-0.30(\mathrm{P}<0.001) \\
\text { Interaction between race and current smoking: } \\
+0.062(\mathrm{P}=0.03) \\
\text { Interaction between educational level and current } \\
\text { smoking: }-0.087(\mathrm{P}=0.004)\end{array}$ \\
\hline
\end{tabular}

$\mathrm{R}^{2}$ for main models: 0.141 to 0.373

AdjB for one SD increase in blood pressure (all individuals)

Men: $0.017(P<0.001)$; women: $0.023(P<0.001)$

Variance explained by traditional risk factors ${ }^{20}$
AdjB for one SD increase in $\mathrm{LDL} / \mathrm{HDL}$ ratio (all individuals)

Men: $0.009(P<0.001)$; women: $0.009(P<0.001)$

AdjB for one SD increase in NC (all individuals)

Men: $0.026(P<0.001)$; women: $0.019(P<0.001)$

AdjB for one SD increase in glycohemoglobin (all individuals)

Men: $0.005(P<0.05)$; women: $0.006(P<0.01)$
CIMT increased with age in all sex and race groups.

CIMT was higher in men than in women.

Black race was associated with higher CIMT values than those of whites. Mixed-race individuals had intermediate values, but these were closer to those found in whites.

CIMT values were significantly and inversely associated with the $\mathrm{CVH}$ score.

After minimizing the influence of the main confounders, there was a small but significant association between systolic blood pressure variability and CIMT values.

Participants with glucose levels between 110 and 125 $\mathrm{mg} / \mathrm{dl}$ in 1998 had higher CIMT values at the ELSA-Brasil baseline than did those with glucose levels $<100 \mathrm{mg} / \mathrm{dl}$. Participants with incident diabetes during follow-up had higher CIMT values at the ELSA-Brasil baseline than did those without incident diabetes.

CIMT values were not associated with HALP after adjustment for confounders.

NC was associated with CIMT but not CAC, thus suggesting that fatty tissues possibly had a local effect.

Individuals with resistant hypertension had higher CIMT values than did those with non-resistant hypertension.

Current and former smokers had higher CIMT values.

The association between current smoking and CIMT was stronger among older individuals, males, non-whites and individuals with lower educational levels.

Less than $40 \%$ of CIMT variance was explained by traditional risk factors.

Pulse pressure, LDL/HDL ratio and neck circumference were the most consistent contributors to the main models, while the association with measurements of glucose metabolism was weaker.

95\% Cl = 95\% confidence interval; AdjB = adjusted beta-coefficient; AdjOR = adjusted odds ratio; AHA = American Heart Association; CIMT = carotid intimamedia thickness; $\mathrm{CVH}=$ cardiovascular health; $\mathrm{HALP}=$ hyperalphalipoproteinemia. $\mathrm{HDL}=$ high-density lipoprotein; $\mathrm{LDL}=$ low-density lipoprotein; $\mathrm{NC}=$ neck circumference; $\mathrm{SD}=$ standard deviation. 
Table 2. ELSA-Brasil studies evaluating the association between carotid intima-media thickness and novel cardiovascular risk factors, and other association studies

\begin{tabular}{|c|c|}
\hline Main aims and associations & Main findings \\
\hline Adiponectin ${ }^{21}$ & $\begin{array}{l}\text { AdjOR for the association with CIMT } \geq 75^{\text {th }} \text { percentile for a } \\
\text { given age, sex and race: } \\
\text { Log-transformed adiponectin: } 0.78 \text { ( } 95 \% \mathrm{Cl}: 0.63-0.97)\end{array}$ \\
\hline Ankle-brachial index ${ }^{22}$ & $\begin{array}{l}\text { Positive likelihood ratios for high CIMT, using: } \\
\text { Highest ankle systolic BP: } 2.79 \text { ( } 95 \% \text { Cl: } 1.50-5.18) \\
\text { Mean ankle systolic BP: } 2.68 \text { (95\% Cl: } 1.74-4.13) \\
\text { Lowest ankle systolic BP: } 1.83 \text { ( } 95 \% \text { Cl: } 1.42-2.36) \\
\text { Negative likelihood ratios for high CIMT, using: } \\
\text { Highest ankle systolic BP: } 0.99 \text { (95\% Cl: } 0.99-1.00) \\
\text { Mean ankle systolic BP: } 0.99 \text { (95\% Cl: } 0.98-1.00) \\
\text { Lowest ankle systolic BP: } 0.98 \text { (95\% Cl: } 0.97-0.99)\end{array}$ \\
\hline Cognitive performance ${ }^{23}$ & $\begin{array}{l}\text { AdjB for delayed word recall test score: }-0.433(95 \% \mathrm{Cl} \text { : } \\
\qquad-0.724 \text { to }-0.142)\end{array}$ \\
\hline Cognitive performance ${ }^{24}$ & $\begin{array}{l}\text { Significant path coefficients for the indirect path } \\
\text { between HRV and the TMT-B via HOMA-IR and CIMT: } \\
\text { Path coefficient for HRV to HOMA-IR: }-0.0942(\mathrm{P}<0.001) \\
\text { Path coefficient for HOMA-IR to CIMT: }+0.0340(\mathrm{P}<0.0001) \\
\text { Path coefficient for CIMT to TMT-B: }+0.1052(\mathrm{P}<0.0001)\end{array}$ \\
\hline Endothelial function ${ }^{25}$ & $\begin{array}{l}\text { AdjB for the reactive hyperemia index: }+0.060(P=0.023) \\
\text { AdjB for the mean basal pulse amplitude: }+0.010(P=0.221)\end{array}$ \\
\hline
\end{tabular}

HIV infection ${ }^{26}$

AdjB for HIV group: 0.004 (95\% Cl: -0.006 to +0.014$)$

Insulin resistance ${ }^{27}$

Mental symptoms ${ }^{28}$

Migraine $^{29}$

Socioeconomic mobility ${ }^{30}$

Socioeconomic status ${ }^{31}$

Subclinical hypothyroidism ${ }^{32}$
AdjB for downward intergenerational mobility (3 or more levels in a 7-level ladder): $+0.013(P=0.04)$

AdjB for stable low social status: $+0.012(P=0.03)$

AdjB for low life course socioeconomic position: Men: $+0.049(95 \% \mathrm{Cl}:+0.020$ to +0.079$)$

Women: $+0.031(95 \% \mathrm{Cl}:+0.001$ to +0.063$)$

AdjB for subclinical hypothyroidism: $+0.010(95 \% \mathrm{Cl}$ : +0.001 to +0.019 )

AdjOR for the association with CIMT $\geq 75^{\text {th }}$ percentile for a One SD increase in HOMA-IR: 1.10 (95\% Cl: 1.04-1.17)

OR for the association with CIMT $\geq 75^{\text {th }}$ percentile for a

$$
\text { given age, sex and race: }
$$

For

AdjB for migraine with aura: -0.01 ( $95 \% \mathrm{Cl}:-0.03$ to +0.01 )

AdjB for migraine without aura: -0.01 ( $95 \% \mathrm{Cl}:-0.02$ to +0.01 )

AdjOR for the association with CIMT $\geq 75^{\text {th }}$ percentile for a given age, sex and race:

\section{Main conclusions}

Low adiponectin levels were associated with CIMT values in this subgroup, after adjustment for multiple confounders (including body mass index).

Positive likelihood ratios for high CIMT ( $\geq 75^{\text {th }}$ percentile) were higher when the highest (compared with mean or lowest) ankle systolic BP was used for ankle-brachial index calculation.

Negative likelihood ratios for high CIMT ( $\geq 75^{\text {th }}$ percentile) were similar for all ankle-brachial index calculation strategies.

CIMT values were inversely associated with performance in memory tests.

Both insulin resistance and CIMT values mediated the association between heart rate variability and performance in the TMT-B.

Endothelial function (according to peripheral arterial tonometry) was inconsistently associated with CIMT.

Endothelial dysfunction and CIMT may represent distinct phenomena or different stages of the atherosclerotic process.

Comparing ELSA-Brasil data with information from individuals with HIV infection (mostly undergoing combined antiretroviral therapy), CIMT values did not differ between groups after adjustment for sociodemographic variables and cardiovascular risk factors.

There was a direct association between insulin resistance and CIMT values, while glucose levels or glycated hemoglobin were not associated with CIMT in the main models. This raised the hypothesis that a direct effect from insulin on atherosclerosis or insulin-promoted medial hypertrophy may be involved.

Intensity and frequency of mental symptoms, along with generalized anxiety disorder and common mental disorder, were associated with higher CIMT values in full models.

Migraine, regardless of the presence of aura symptoms, was not associated with CIMT values after adjustment for confounders. Downward intergenerational mobility was associated with higher CIMT values, and the more intense the downward mobility was, the more intense this association also was. For intragenerational mobility, individuals with stable low social status had higher CIMT values than did those with stable high social status. CIMT values were positively associated with more prolonged exposure to low socioeconomic status (cumulative lifetime socioeconomic position).

Subclinical hypothyroidism was associated with CIMT values after adjustment for major confounders.

Subclinical hypothyroidism: 1.30 (95\% Cl: 1.06-1.59)

95\% Cl = 95\% confidence interval; AdjB = adjusted beta-coefficient; AdjOR = adjusted odds ratio; BP = blood pressure; CIMT = carotid intima-media thickness. HOMA-IR = homeostasis model assessment - insulin resistance; $\mathrm{OR}=$ odds ratio; SD = standard deviation; TMT-B: trail-making test B. 
four different classes of antihypertensive medication) had higher CIMT values than did those with non-resistant hypertension, thus suggesting that a dose-response relationship existed between BP and CIMT. However, this association is not limited to individuals using antihypertensives. Recently, Ribeiro et al. ${ }^{14}$ analyzed the association between CIMT values and the patterns in the ten BP measurements that were made during the six-hour baseline visit among ELSA-Brasil participants who were not using antihypertensive drugs. They found that both the central trend (as a weighted mean) of the systolic BP measurements and the systolic BP variability (standard deviation of BP measurements) were associated with CIMT values, after minimizing the influence of other traditional cardiovascular risk factors. This finding suggests that high short-term BP variability has a role in CIMT values and, potentially, in atherosclerosis.

Sitnik et al. ${ }^{15}$ analyzed data from a subset of ELSA-Brasil participants at the São Paulo investigation site $(\mathrm{N}=1536)$ who had participated in a workplace screening assessment in 1998 and did not have diabetes at that time. One aim of that study was to evaluate whether glucose levels in 1998 or incident diabetes between assessments would predict CIMT levels at the ELSA-Brasil baseline, 10 to 12 years later. They found that participants with incident diabetes between assessments had higher CIMT values at the ELSABrasil baseline. Individuals with glucose levels between $110 \mathrm{mg} / \mathrm{dl}$ and $125 \mathrm{mg} / \mathrm{dl}$ in 1998 also had higher CIMT values at the ELSABrasil baseline than did those with glucose levels below $100 \mathrm{mg} / \mathrm{dl}$, although this association lost significance when individuals who developed diabetes were excluded ( $\mathrm{P}=0.093)$.

Elevated high-density lipoprotein cholesterol (HDL-c) levels are typically considered to provide a protective cardiovascular profile. However, there is evidence linking hyperalphalipoproteinemia (HALP), a condition related to very high HDL-c levels, to higher cardiovascular risk, ${ }^{38}$ probably due to abnormal HDL-c activity. Using the very large CIMT dataset from the ELSA-Brasil baseline, Laurinavicius et al. ${ }^{16}$ found no evidence of higher CIMT values in individuals with HDL-c levels above $90 \mathrm{mg} / \mathrm{dl}$, compared with those with normal HDL levels, in adjusted models.

Recently, Kianoush et al. ${ }^{19}$ further explored the association between smoking status and a set of inflammatory and atherosclerotic markers, including CIMT. Besides noting the fact that current and former smokers have higher CIMT values, these authors also suggested that this association is stronger among individuals over 50 years of age, males, non-whites and individuals with lower educational levels. They also found an association between secondhand smoking and CIMT values in models adjusted for age, sex, race and other cardiovascular risk factors. However, this association only maintained borderline significance after adjustment for participants' smoking status and pack-years $(\mathrm{P}=0.06)$.

\section{CIMT and novel cardiovascular risk factors or other conditions}

One of the greatest strengths of the ELSA-Brasil cohort is that it enables studies on the effects of social variables on clinical (biological) characteristics. Two ELSA-Brasil studies have focused on the relationship between socioeconomic position and trajectory and CIMT. Camelo et al. ${ }^{31}$ evaluated the relationship between CIMT and socioeconomic position over the course of life, as measured by the participants' jobs in their first and current occupations. They found that the association between CIMT and socioeconomic position over the course of life followed a cumulative model, in which CIMT levels were positively associated with more time spent performing jobs of low socioeconomic level. High work stress and passive work were not associated with CIMT, regardless of socioeconomic level. A different analytical strategy was adopted by Guimarães et al., ${ }^{30}$ who also analyzed intergenerational social mobility. In this study, in comparing the change in occupational social class of the head of the household between the participant's occupation at the time of starting to work and his or her current occupation, the participants with downward mobility had higher CIMT values. Moreover, the association was more intense for individuals with greater downward mobility.

Endothelial dysfunction is an important phase of the atherosclerotic process ${ }^{39}$ that can be measured using peripheral arterial tonometry (PAT). In a subset of participants at the Minas Gerais investigation site, Lemos et al. ${ }^{25}$ evaluated the association between PAT measurements and CIMT values. These authors did not find any consistent associations, thus suggesting that information from PAT and CIMT may be complementary and may represent different phenomena or stages of the atherosclerotic process.

Novel information about CIMT pathophysiology and interpretation resulted from two recent ELSA-Brasil studies. Santos et al. ${ }^{27}$ showed that insulin resistance was more strongly associated with CIMT values than with glucose levels, thus suggesting that a hormonal effect on CIMT may exist. This may arise through a direct effect from insulin on the pathogenesis of atherosclerosis. On the other hand, hyperinsulinemia (consequential to insulin-resistant states) may also cause medial hypertrophy, which is included in the intima-media complex. This second hypothesis is further supported by findings from de Almeida-Pititto et al., ${ }^{21}$ who studied the association between CIMT values and serum adiponectin in a subsample of 687 ELSA-Brasil participants in São Paulo aged 35 to 54 years, without diabetes or cardiovascular disease at baseline. Adiponectin also inhibits vascular smooth muscle cell proliferation, and these authors found higher CIMT values in individuals with lower adiponectin levels. In addition, both higher insulin resistance and lower adiponectin levels have been correlated with visceral (including perivascular) adiposity, and we speculate that these may be potential mediators because of their association with higher cardiovascular risk. 
Mental disorders and clinical cardiovascular disease are two frequently coexistent conditions, with putative bidirectional causality between them. ${ }^{40}$ The ELSA-Brasil baseline forms a good scenario for studying the association between subclinical atherosclerosis and mental disorders, since this baseline assessment included a validated Portuguese-language version of the Clinical Interview Schedule Revised (CIS-R) questionnaire. CIS-R addresses non-psychotic mental symptoms and enables diagnoses in accordance with the tenth version of the International Classification of Diseases (ICD10). Santos et al. ${ }^{28}$ found that higher CIS-R scores (reflecting more frequent and/or more intense non-psychotic mental symptoms) and a diagnosis of generalized anxiety disorder were associated with higher CIMT values in adjusted models.

Vascular disease is a well-known cause of dementia, but there is conflicting evidence for the association between subclinical carotid atherosclerosis and cognitive decline. Suemoto et al. ${ }^{23}$ studied the association between CIMT and cognitive performance among individuals without a medical history of stroke at the ELSABrasil baseline. These authors found that there was an association between poorer performance in a memory test (the delayed word recall test) and higher CIMT values, after adjustment for sociodemographic variables, cardiovascular risk factors, diagnosed depression and thyroid function. In addition, Kemp et al. ${ }^{24}$ found that CIMT mediates the association between heart rate variability (an index of cardiac autonomic function) and performance in the trail-making test $\mathrm{B}$ (an executive cognitive function test), thus further supporting the link between cardiovascular diseases and cognitive performance. One strength of these analyses was that they included a large number of younger adults, a population that had been poorly studied previously.

One continuing controversy is the association between migraine and cardiovascular diseases. Goulart et al. ${ }^{29}$ studied the association between a diagnosis of migraine (defined using a validated questionnaire based on the International Headache Society criteria) and subclinical atherosclerosis at the ELSA-Brasil baseline among participants at the São Paulo investigation site. There were no significant associations between migraine (with or without aura) and CIMT values. These results were consistent when subclinical atherosclerosis was defined according to coronary artery calcium (CAC) scores.

Hypothyroidism is associated with a poorer cardiovascular profile, ${ }^{41}$ but the effects of subclinical thyroid dysfunction on atherosclerosis is a matter for debate. Peixoto de Miranda et al. ${ }^{32}$ studied the association between CIMT values and subclinical hypothyroidism (high thyroid-stimulating hormone with normal free thyroxine levels) at the ELSA-Brasil baseline. In adjusted models, individuals with subclinical hypothyroidism at the baseline had significantly higher CIMT values than did those with normal thyroid function.
Miname et al. ${ }^{22}$ compared different protocols for calculating the ankle-brachial index ( $\mathrm{ABI}$, a measurement of peripheral artery disease) at the ELSA-Brasil baseline. ABI is based on the ratio of systolic $\mathrm{BP}$ measured at the ankle divided by systolic $\mathrm{BP}$ measured in the arm. These authors found that when the highest ankle systolic BP was used for ABI calculation, the association with CIMT values was stronger than if they used the mean or lowest ankle systolic BP.

The ELSA-Brasil CIMT data were also used to evaluate the atherosclerotic burden associated with HIV infection. Pacheco et al. ${ }^{26}$ compared data from HIV-positive patients who were followed in a cohort in Rio de Janeiro, HIV-negative friends of these subjects, and ELSA-Brasil participants. These authors reported that there were no significant associations between HIV infection status and CIMT values in adjusted models.

\section{ELSA-Brasil Visit 3 and perspectives}

The third onsite assessment (Visit 3) of the ELSA-Brasil participants began in early 2017, and is expected to end in 2018 . New questionnaires, clinical and laboratory examinations, and CIMT and femoral IMT are included in the Visit 3 protocol. These new data will make it possible to study baseline characteristics that have influenced CIMT progression over an eight-year period. Furthermore, concomitant evaluation of the carotid and femoral arteries may enable greater understanding of how associations with IMT values vary in different arterial beds. Along these lines, some studies ${ }^{42-44}$ have shown that cardiovascular risk factors associated with increased CIMT are associated with IMT values at other sites, such as the femoral arteries, but the magnitudes of these associations are heterogeneous. The current approach will allow deeper exploration of relevant scientific questions raised in previous ELSA-Brasil published data, such as the potential paracrine effect of NC on CIMT, for example. On the other hand, other factors that are only weakly associated with CIMT may be more important for determining femoral IMT values.

ELSA-Brasil was designed as a cohort study. One very important research question that longitudinal ELSA-Brasil data may help to answer concerns the extent to which CIMT values and CIMT progression may predict cardiovascular events. Most current data suggest that CIMT values have higher importance than does CIMT progression in cardiovascular risk prediction. ${ }^{3,45}$ However, conflicting data exists, ${ }^{46,47}$ at least partially due to different study populations and heterogeneous CIMT protocols. In addition, plaque analyses (including total plaque area information) for the common carotid, carotid bulb and internal carotid arteries at the baseline are currently underway. Cross-sectional and longitudinal analyses using these data may be available soon. 


\section{CONCLUSIONS}

ELSA-Brasil is one of the largest studies with CIMT data. Most published studies have focused on associations with traditional and novel cardiovascular risk factors. Analyses on the ELSABrasil data have also led to important insights regarding CIMT interpretation and physiology. In addition to the contributions highlighted here that have already made to this field, new data gathered during the ongoing third onsite assessment will enable investigation of substantially new research questions.

\section{REFERENCES}

1. Touboul PJ, Hennerici MG, Meairs S, et al. Mannheim carotid intimamedia thickness and plaque consensus (2004-2006-2011). An update on behalf of the advisory board of the 3rd, 4th and 5th watching the risk symposia, at the 13th, 15th and 20th European Stroke Conferences, Mannheim, Germany, 2004, Brussels, Belgium, 2006, and Hamburg, Germany, 2011. Cerebrovasc Dis. 2012;34(4):290-6.

2. Heiss G, Sharrett AR, Barnes R, et al. Carotid atherosclerosis measured by B-mode ultrasound in populations: associations with cardiovascular risk factors in the ARIC study. Am J Epidemiol. 1991;134(3):250-6.

3. Lorenz MW, Markus HS, Bots ML, Rosvall M, Sitzer M. Prediction of clinical cardiovascular events with carotid intima-media thickness: a systematic review and meta-analysis. Circulation. 2007;115(4):459-67.

4. Zanchetti A, Bond MG, Hennig M, et al. Calcium antagonist lacidipine slows down progression of asymptomatic carotid atherosclerosis: principal results of the European Lacidipine Study on Atherosclerosis (ELSA), a randomized, double-blind, long-term trial. Circulation. 2002;106(19):2422-7.

5. Aquino EM, Barreto SM, Bensenor IM, et al. Brazilian Longitudinal Study of Adult Health (ELSA-Brasil): objectives and design. Am J Epidemiol. 2012;175(4):315-24.

6. Schmidt MI, Duncan BB, Mill JG, et al. Cohort Profile: Longitudinal Study of Adult Health (ELSA-Brasil). Int J Epidemiol. 2015;44(1):68-75.

7. Howard G, Sharrett AR, Heiss G, et al. Carotid artery intimal-medial thickness distribution in general populations as evaluated by B-mode ultrasound. ARIC Investigators. Stroke. 1993;24(9):1297-304.

8. Folsom AR, Kronmal RA, Detrano RC, et al. Coronary artery calcification compared with carotid intima-media thickness in the prediction of cardiovascular disease incidence: the Multi-Ethnic Study of Atherosclerosis (MESA). Arch Intern Med. 2008;168(12):1333-9.

9. Rundek T, Blanton SH, Bartels S, et al. Traditional risk factors are not major contributors to the variance in carotid intima-media thickness. Stroke. 2013;44(8):2101-8.

10. Bots ML, Hofman A, Grobbee DE. Increased common carotid intimamedia thickness. Adaptive response or a reflection of atherosclerosis? Findings from the Rotterdam Study. Stroke. 1997;28(12):2442-7.

11. Lotufo PA. Construção do Estudo Longitudinal de Saúde do Adulto (ELSA-Brasil) Setting up the Longitudinal Study for Adult Health (ELSABrasil). Rev Saúde Pública. 2013;47 suppl 2:3-9.
12. Santos IS, Bittencourt MS, Oliveira IR, et al. Carotid intima-media thickness value distributions in the Brazilian Longitudinal Study of Adult Health (ELSA-Brasil). Atherosclerosis. 2014;237(1):227-35.

13. Santos IS, Goulart AC, Pereira AC, Lotufo PA, Benseñor IM. Association between Cardiovascular Health Score and Carotid Intima-Media Thickness: Cross-Sectional Analysis of the Brazilian Longitudinal Study of Adult Health (ELSA-Brasil) Baseline Assessment. J Am Soc Echocardiogr. 2016;29(12):1207-16.e4

14. Ribeiro AH, Lotufo PA, Fujita A, et al. Association Between Short-Term Systolic Blood Pressure Variability and Carotid Intima-Media Thickness in ELSA-Brasil Baseline. Am J Hypertens. 2017;30(10):954-60.

15. Sitnik D, Santos IS, Goulart AC, et al. Fasting glucose levels, incident diabetes, subclinical atherosclerosis and cardiovascular events in apparently healthy adults: A 12-year longitudinal study. Diab Vasc Dis Res. 2016;13(6):429-37.

16. Laurinavicius AG, Santos IS, Santos RD, et al. Extremely elevated HDLcholesterol levels are not associated with increased carotid intima-media thickness: data from ELSA Brasil. J Clin Lipidol. 2016;10(4):898-904.e1.

17. Baena CP, Lotufo PA, Santos IS, et al. Neck circumference is associated with carotid intimal-media thickness but not with coronary artery calcium: Results from The ELSA-Brasil. Nutr Metab Cardiovasc Dis. 2016;26(3):216-22.

18. Lotufo PA, Pereira AC, Vasconcellos PS, et al. Resistant hypertension: risk factors, subclinical atherosclerosis, and comorbidities among adults-the Brazilian Longitudinal Study of Adult Health (ELSA-Brasil). J Clin Hypertens (Greenwich). 2015;17(1):74-80.

19. Kianoush S, Yakoob MY, Al-Rifai M, et al. Associations of Cigarette Smoking With Subclinical Inflammation and Atherosclerosis: ELSA-Brasil (The Brazilian Longitudinal Study of Adult Health). J Am Heart Assoc. 2017;6(6). pi: e005088.

20. Santos IS, Alencar AP, Rundek T, et al. Low Impact of Traditional Risk Factors on Carotid Intima-Media Thickness: The ELSA-Brasil Cohort. Arterioscler Thromb Vasc Biol. 2015;35(9):2054-9.

21. de Almeida-Pititto B, Ribeiro-Filho FF, Santos IS, et al. Association between carotid intima-media thickness and adiponectin in participants without diabetes or cardiovascular disease of the Brazilian Longitudinal Study of Adult Health (ELSA-Brasil). Eur J Prev Cardiol. 2017;24(2):116-22.

22. Miname M, Bensenor IM, Lotufo PA. Different methods of calculating ankle-brachial index in mid-elderly men and women: the Brazilian Longitudinal Study of Adult Health (ELSA-Brasil). Braz J Med Biol Res. 2016;49(12):e5734.

23. Suemoto CK, Santos IS, Bittencourt MS, et al. Subclinical carotid artery atherosclerosis and performance on cognitive tests in middleaged adults: Baseline results from the ELSA-Brasil. Atherosclerosis. 2015;243(2):510-5.

24. Kemp AH, López SR, Passos VMA, et al. Insulin resistance and carotid intima-media thickness mediate the association between resting-state heart rate variability and executive function: A path modelling study. Biol Psychol. 2016;117:216-24. 
25. Lemos SP, Passos VM, Brant LC, et al. Inconsistent Correlation Between Carotid Artery Intima-Media Thickness and Peripheral Arterial Tonometry: Brazilian Longitudinal Study of Adult Health (ELSA-Brasil). Medicine (Baltimore). 2015;94(33):e1403.

26. Pacheco AG, Grinsztejn B, Fonseca Mde J, et al. HIV Infection Is Not Associated with Carotid Intima-Media Thickness in Brazil: A Cross-Sectional Analysis from the INI/ELSA-Brasil Study. PLoS One. 2016;11(7):e0158999.

27. Santos IS, Bittencourt MS, Goulart AC, et al. Insulin resistance is associated with carotid intima-media thickness in non-diabetic subjects. A crosssectional analysis of the ELSA-Brasil cohort baseline. Atherosclerosis. 2017;260:34-40.

28. Santos IS, Goulart AC, Brunoni AR, et al. Anxiety and depressive symptoms are associated with higher carotid intima-media thickness. Cross-sectional analysis from ELSA-Brasil baseline data. Atherosclerosis. 2015;240(2):529-34.

29. Goulart AC, Santos IS, Bittencourt MS, Lotufo PA, Benseñor IM. Migraine and subclinical atherosclerosis in the Brazilian Longitudinal Study of Adult Health (ELSA-Brasil). Cephalalgia. 2016;36(9):840-8.

30. Guimarães JM, Clarke P, Tate D, et al. Social mobility and subclinical atherosclerosis in a middle-income country: Association of intra- and inter-generational social mobility with carotid intima-media thickness in the Brazilian Longitudinal Study of Adult Health (ELSA-Brasil). Soc Sci Med. 2016;169:9-17.

31. Camelo LV, Giatti L, Chor D, et al. Associations of life course socioeconomic position and job stress with carotid intima-media thickness. The Brazilian Longitudinal Study of Adult Health (ELSA-Brasil). Soc Sci Med. 2015;141:91-9.

32. Peixoto de Miranda ÉJ, Bittencourt MS, Pereira AC, et al. Subclinical hypothyroidism is associated with higher carotid intima-media thickness in cross-sectional analysis of the Brazilian Longitudinal Study of Adult Health (ELSA-Brasil). Nutr Metab Cardiovasc Dis. 2016;26(10):915-21.

33. Mill JG, Pinto K, Griep RH, et al. Afericoes e exames clinicos realizados nos participantes do ELSA-Brasil [Medical assessments and measurements in ELSA-Brasil]. Rev Saúde Pública. 2013;47(supl 2):54-62.

34. Freire CMV, Alcantara ML, Santos SN, et al. Recomendação para a Quantificação pelo Ultrassom da Doença Aterosclerótica das Artérias Carótidas e Vertebrais: Grupo de Trabalho do Departamento de Imagem Cardiovascular da Sociedade Brasileira de Cardiologia - DIC - SBC. Arq Bras Cardiol: Imagem Cardiovasc. 2015;28(no. especial):e1-e64. Available from: http://departamentos.cardiol.br/dic/publicacoes/ revistadic/revista/2015/portugues/Numero_Especial/vol28_artigo\%20 especial_port.pdf. Accessed in 2017 (Oct 31).

35. Stein JH, Korcarz CE, Hurst RT, et al. Use of carotid ultrasound to identify subclinical vascular disease and evaluate cardiovascular disease risk: a consensus statement from the American Society of Echocardiography Carotid Intima-Media Thickness Task Force. Endorsed by the Society for Vascular Medicine. J Am Soc Echocardiogr. 2008;21(2):93-111; quiz 189-90.
36. Lloyd-Jones DM, Hong Y, Labarthe D, et al. Defining and setting national goals for cardiovascular health promotion and disease reduction: the American Heart Association's strategic Impact Goal through 2020 and beyond. Circulation. 2010;121(4):586-613.

37. Bauer M, Delaney JA, Möhlenkamp S, et al. Comparison of factors associated with carotid intima-media thickness in the Multi-ethnic Study of Atherosclerosis (MESA) and the Heinz Nixdorf Recall Study (HNR). J Am Soc Echocardiogr. 2013;26(6):667-73.

38. Hirano K, Yamashita S, Kuga Y, et al. Atherosclerotic disease in marked hyperalphalipoproteinemia. Combined reduction of cholesteryl ester transfer protein and hepatic triglyceride lipase. Arterioscler Thromb Vasc Biol. 1995;15(11):1849-56.

39. Bentzon JF, Otsuka F, Virmani R, Falk E. Mechanisms of plaque formation and rupture. Circ Res. 2014;114(12):1852-66.

40. Bivanco-Lima D, Santos IS, Vannucchi AMC, Ribeiro MCA. Risco cardiovascular em indivíduos com depressão [Cardiovascular risk in individuals with depression]. Rev Assoc Med Bras (1992). 2013;59(3):298-304.

41. Jabbar A, Pingitore A, Pearce SH, et al. Thyroid hormones and cardiovascular disease. Nat Rev Cardiol. 2017;14(1):39-55.

42. Pasterkamp G, Schoneveld AH, Hillen B, et al. Is plaque formation in the common carotid artery representative for plaque formation and luminal stenosis in other atherosclerotic peripheral arteries? A post mortem study. Atherosclerosis. 1998;137(1):205-10.

43. Fernández-Friera L, Peñalvo JL, Fernández-Ortiz A, et al. Prevalence, Vascular Distribution, and Multiterritorial Extent of Subclinical Atherosclerosis in a Middle-Aged Cohort: The PESA (Progression of Early Subclinical Atherosclerosis) Study. Circulation. 2015;131(24):2104-13.

44. Laclaustra M, Casasnovas JA, Fernández-Ortiz A, et al. Femoral and Carotid Subclinical Atherosclerosis Association with Risk Factors and Coronary Calcium: The AWHS Study. J Am Coll Cardiol. 2016;67(11):1263-74.

45. Lorenz MW, Polak JF, Kavousi M, et al. Carotid intima-media thickness progression to predict cardiovascular events in the general population (the PROG-IMT collaborative project): a meta-analysis of individual participant data. Lancet. 2012;379(9831):2053-62.

46. Baldassarre D, Veglia F, Hamsten A, et al. Progression of carotid intimamedia thickness as predictor of vascular events: results from the IMPROVE study. Arterioscler Thromb Vasc Biol. 2013;33(9):2273-9.

47. Polak JF, Pencina MJ, O'Leary DH, D'Agostino RB. Common carotid artery intima-media thickness progression as a predictor of stroke in multi-ethnic study of atherosclerosis. Stroke. 2011;42(11):3017-21.

Sources of funding: The ELSA-Brasil baseline study was supported by the Brazilian Ministry of Health (Science and Technology Department) and the Brazilian Ministry of Science and Technology (Financiadora de Estudos e Projetos and Conselho Nacional de Desenvolvimento Científico e Tecnológico, CNPq) (grant numbers 01060010.00 RS, 0106 
0212.00 BA, 01060300.00 ES, 01060278.00 MG, 01060115.00 SP and

$01060071.00 \mathrm{RJ})$. Itamar de Souza Santos is a recipient of a research

fellowship from the CNPq (grant number 302364/2016-1) and a research grant from the Fundação de Amparo à Pesquisa do Estado de São Paulo

(FAPESP; grant number 2015/17013-3)

Conflicts of interest:The authors report that there were no conflicts of interest

Date of first submission: August 30, 2017

Last received: October 6, 2017

Accepted: October 14, 2017

Address for correspondence:

Itamar de Souza Santos

Centro de Pesquisa Clínica e Epidemiológica do Hospital Universitário da

Universidade de São Paulo

Av. Professor Lineu Prestes, 2.565 - 3ㅇandar

São Paulo (SP) - Brasil

CEP 05508-000

Tel. (+55 11) 3091-9300

E-mail: itamarss@usp.br 